\title{
Is Acculturation Related to Obesity in Hispanic/Latino Adults? Results from the Hispanic Community Health Study/Study of Latinos
}

\author{
Carmen R. Isasi, ${ }^{1}$ Guadalupe X. Ayala, ${ }^{2}$ Daniela Sotres-Alvarez, ${ }^{3}$ Hala Madanat, ${ }^{2}$ \\ Frank Penedo, ${ }^{4}$ Catherine M. Loria, ${ }^{5}$ John P. Elder, ${ }^{2}$ Martha L. Daviglus, ${ }^{6}$ Janice Barnhart, ${ }^{1}$ \\ Anna Maria Siega-Riz, ${ }^{7}$ Linda Van Horn, ${ }^{8}$ and Neil Schneiderman' \\ ${ }^{1}$ Department of Epidemiology \& Population Health, Albert Einstein College of Medicine, Bronx, NY 10461, USA \\ ${ }^{2}$ Division of Health Promotion and Behavioral Science, Graduate School of Public Health, San Diego State University and \\ the Institute for Behavioral and Community Health, San Diego State University Research Foundation, San Diego, CA 92123, USA \\ ${ }^{3}$ Collaborative Studies Coordinating Center, Department of Biostatistics, Gillings School of Global Public Health, \\ University of North Carolina at Chapel Hill, Chapel Hill, NC 27514, USA \\ ${ }^{4}$ Department of Medical Social Sciences, Feinberg School of Medicine, Northwestern University, Chicago, IL 60611, USA \\ ${ }^{5}$ Division of Cardiovascular Sciences, National Heart, Lung, and Blood Institute, Bethesda, MD 20817, USA \\ ${ }^{6}$ Institute for Minority Health Research, University of Illinois at Chicago, Chicago, IL 60612, USA \\ ${ }^{7}$ Departments of Epidemiology and Nutrition, Gillings School of Global Public Health, \\ University of North Carolina at Chapel Hill, Chapel Hill, NC 27599, USA \\ ${ }^{8}$ Department of Preventive Medicine, Feinberg School of Medicine, Northwestern University, Chicago, IL 60126, USA \\ ${ }^{9}$ The Behavioral Research Center, Department of Psychology, University of Miami, Coral Gables, FL 33124, USA
}

Correspondence should be addressed to Carmen R. Isasi; carmen.isasi@einstein.yu.edu

Received 19 August 2014; Accepted 11 March 2015

Academic Editor: Denis Richard

Copyright (c) 2015 Carmen R. Isasi et al. This is an open access article distributed under the Creative Commons Attribution License, which permits unrestricted use, distribution, and reproduction in any medium, provided the original work is properly cited.

Background. The study examined the association of obesity with acculturation in a large and diverse sample of US Hispanic/Latino adults. Methods. The Hispanic Community Health Study (HCHS)/Study of Latinos (SOL) is a community-based cohort study of Hispanic/Latino adults aged 18-74 years $(N=16,415)$ from four urban areas. Height and weight were directly measured using a standardized protocol. Acculturation was assessed by the Short Acculturation Scale for Hispanics (SASH). Other immigration related variables included place of birth, length of residency in the US, and age at immigration. Odds ratios were calculated to assess the association of overweight, moderate obesity, and extreme obesity $\left(\geq 40 \mathrm{~kg} / \mathrm{m}^{2}\right)$ with acculturation and sociodemographic variables. Results. The prevalence of obesity was $42.4 \%$ for women and $36.5 \%$ for men and varied by field center and Hispanic/Latino background. The strongest predictor of moderate and extreme obesity was length of residency in mainland US. This association was consistent across Hispanic/Latino backgrounds. Acculturation was not significantly associated with obesity. Discussion. The burden of obesity is high among Hispanic/Latino adults. The study findings suggest that prolonged exposure to the environments in these communities, rather than acculturation, is an important risk factor for obesity in this population.

\section{Introduction}

Hispanics living in the US are disproportionately represented in the obesity epidemic. Data from National Health and Nutrition Examination Survey (NHANES) 2009-2010 showed that the prevalence of obesity among Hispanic adults, primarily Mexican Americans, is higher than non-Hispanic whites, with significant increases over the last decade for Mexican Americans [1]. Despite their diversity in origins and culture [2], national survey data do not present a clear picture of the burden of obesity and its risk factors among the various Hispanic groups other than those of Mexican 
origin. Emerging evidence suggests that country of origin may have important implications for understanding disease risks [3]. In addition, research has shown that being born in the US and longer duration of residency are associated with a higher prevalence of obesity [4], suggesting that acculturation may play a role in the development of obesity. Acculturation research examines the extent to which continuous and first-hand contact with a new dominant culture that is different from one's culture of origin is associated with individual behavior change $[5,6]$. However, few studies have examined the association of acculturation with obesity in a diverse sample of Hispanics from different backgrounds or nationalities.

In this paper we examined the association between acculturation and obesity in the largest study of Hispanic/Latino adults living in the US. We also examined differences in the prevalence of obesity by Hispanic/Latino background and socioeconomic status indicators. Furthermore, because the prevalence of extreme obesity has risen in the US, particularly among minority groups [7], and because individuals with extreme obesity have higher CVD risk and mortality [8], we also examined the burden of extreme obesity and the sociodemographic risk factors associated with the prevalence of this condition in this diverse sample of Hispanic/Latino adults.

\section{Methods}

2.1. Participants. HCHS/SOL is a community based cohort study of 16,415 self-identified Hispano/Latino individuals aged 18-74 recruited from 2008 to 2011 in four US field centers (Chicago, IL; Miami, FL; Bronx, NY; San Diego, CA). HCHS/SOL participants include those who self-identified their background as Central American $(n=1,732)$, Cuban $(n=2,348)$, Dominican $(n=1,473)$, Mexican $(n=6,472)$, Puerto Rican $(n=2,728)$, and South American $(n=1,072)$. HCHS/SOL goals are to describe the prevalence of risk and protective factors for various chronic conditions (e.g., cardiovascular disease (CVD), diabetes, and pulmonary disease) and to quantify all-cause mortality, fatal and nonfatal CVD and pulmonary disease, and pulmonary disease exacerbation over time [9].

The sample design and cohort selection have been previously described [10]. Briefly, a stratified two-stage area probability sample of household addresses was selected in each of the four field centers. The HCHS/SOL target population is defined as all noninstitutionalized Hispanic/Latino adults aged 18-74 years and residing in the defined geographical areas (census block groups) across the four participating field centers. HCHS/SOL participants were selected using a probability sampling design within these areas to provide a representative sample of the target population. The study oversampled 45-74-year-olds to ensure that the sample size was large enough for analysis of cardiovascular events. Because participants were selected with unequal probabilities, sampling weights were generated to reflect the probabilities of selection at each stage. The study was approved by each field center's institutional review board.
Of the 16,415 participants enrolled in the study, 149 participants were excluded from the analysis because of missing Hispanic/Latino background ( $n=87)$, BMI $(n=53)$, or age ineligible $(>74$ years) at baseline $(n=9)$, leaving an analytic sample of 16,266 participants.

\subsection{Study Measures}

2.2.1. Overweight and Obesity. Height and weight were obtained at each field center clinic using standardized protocols. Height was measured with a wall stadiometer (SECA 222, Germany) and weight was obtained with a digital scale (Tanita Body Composition Analyzer, TBF 300, Japan). Body mass index was calculated as weight in kilograms divided by height in meters squared. Overweight was defined as BMI 25.0 to $29.9 \mathrm{~kg} / \mathrm{m}^{2}$ and obesity as a BMI of $30.0 \mathrm{~kg} / \mathrm{m}^{2}$ or higher. Using NHLBI guidelines obese participants were further classified as having moderate obesity (obesity classes I and II, BMI 30.0 to $39.9 \mathrm{~kg} / \mathrm{m}^{2}$ ) or extreme obesity (obesity class III, BMI $\geq 40 \mathrm{~kg} / \mathrm{m}^{2}$ ) [11].

2.2.2. Measures of Immigration History. Based on participants' place of birth report, they were classified as either born or not outside the US mainland (50 states). Hence, those born in Puerto Rico or other US territories were also classified as born outside US mainland to better reflect their migration and acculturation patterns. Foreign born individuals were asked the number of years lived in the mainland US. Age at immigration was calculated from their reported age and the duration of residence (years) in mainland US.

2.2.3. Measures of Acculturation. Two indices of acculturation were considered. Participants completed an abbreviated 10-item version of Marin's Short Acculturation Scale for Hispanics (SASH) [12], with items related to language preference, media preference, and social affiliations. Two factors were identified (SASH language use, $\alpha=0.92$, and SASH ethnic social relations, $\alpha=0.73$ ), and these two subscales were analyzed separately in the models. Dietary acculturation asked if the types of foods participants usually ate were from Hispanic/Latino or American origin on a 5-level Likert scale ranging from " $1=$ mostly Hispanic" to " $5=$ mostly American foods."

2.2.4. Sociodemographic Variables. Participants were asked their Hispanic/Latino background (Central American, Cuban, Dominican, Mexican, Puerto Rican, South American, and other/mixed), date of birth, gender, household income, and educational attainment.

2.3. Statistical Analysis. Age-adjusted or age-/sex-adjusted prevalence of overweight and obesity was estimated to describe the target population by sex, sociodemographic, and immigration and acculturation characteristics using designbased estimates from survey linear regression models [13]. To facilitate comparisons to NHANES, we also report the prevalence of obesity age-standardized to the year 2010 US population by sex and Hispanic/Latino background. To study 
the association (odds ratio) of Hispanic/Latino background, sociodemographic, immigration history (place of birth, years living in mainland US), and acculturation variables (language use and social relations SASH subscales, dietary acculturation) with BMI categories, we used survey generalized logit regression models. To assess the effect modification by Hispanic/Latino background we included separately the interaction term between each acculturation measure and background. We first tested whether this association was confounded by field center because in HCHS/SOL they are highly collinear due to the fact that people with specific Hispanic/Latino backgrounds tend to concentrate in specific geographic areas (Dominicans are predominantly in the Bronx, Cubans in Miami, and San Diego site mostly has Mexicans). Specifically, we tested the effect of field center $(3 \mathrm{df})$ at an inflated significance level of 0.15 to be more conservative since some of the effect for field center can be absorbed by the estimates for background resulting in loss of power. If field center was not significant at this threshold, then this was interpreted as no evidence in which the effect of Hispanic/Latino background is confounded by field center and the effect of background can be directly assessed. In contrast, if field center was found to be significant, then we cannot know whether identified differences are due to Hispanic/Latino background or field center, and further analyses were conducted to understand these differences. We created a 17-level nominal variable formed from the cross classification of field center and Hispanic/Latino background including only cells where the sample size for the cross classification was greater than 100. Thus, we included the interaction between Hispanic/Latino background and field center without cells with very low sample size. We then used this 17-level covariate to fit a model to calculate the OR between field centers by Hispanic/Latino background. Lastly, among those born outside the US, we studied the association (odds ratio) of age at immigration with BMI group. All analyses accounted for the complex survey design and sampling weights (which include nonresponse adjustment relative to the sampling frame) using SAS version 9.3 corresponding procedures and SAS-callable SUDAAN version 10.

\section{Results}

Sixty percent of the sample was females $(N=9747)$. The mean age was $45.8 \pm 13.9$ years with Central Americans being the youngest group $(44.5 \pm 13.4)$ and Cubans the oldest $(48.9 \pm 13.2) ; 2,660$ participants were in the 18-29-year age group, 2,371 in the 30-39, 4,178 in the 40-49, 4,299 in the $50-59$, and 2,758 in the $60-74$ age group. The majority of participants were first generation immigrants $(81.0 \%)$ and of low socioeconomic status $38.2 \%$ did not graduate from high school, and $48.2 \%$ had an annual household income of $<\$ 20,000)$.

3.1. Prevalence of Overweight and Obesity. The overall ageadjusted prevalence of obesity (BMI $\geq 30 \mathrm{~kg} / \mathrm{m}^{2}$ ) was $42.4 \%$ (95\% CI 40.7, 44.0) for women and $36.5 \%$ for men (95\% CI $34.7,38.3)$. The overall age-adjusted prevalence of extreme obesity (BMI $\geq 40 \mathrm{~kg} / \mathrm{m}^{2}$ ) was $7.4 \%$ in women and $3.7 \%$ in men. There appeared to be differences in the prevalence of obesity. The Bronx presented the largest prevalence of obesity (44.7\%; 95\% CI 42.1-47.2), followed by Chicago (42.1\%; 95\% CI 39.9, 44.4), San Diego (38.6\%; 95\% CI 35.4, 41.7), and Miami (36.7\%; 95\% CI 34.9, 38.6). However, some of the confidence intervals overlapped. Differences in extreme obesity followed the same pattern; the Bronx had the highest prevalence of extreme obesity $(7.1 \%$; 95\% CI 5.6, 8.6), followed by Chicago (5.5\%; 95\% CI 4.7, 6.4), San Diego (5.0\%; 95\% CI 3.8, 6.2), and Miami (4.2\%; 95\% CI 3.4, 5.0). The ageadjusted prevalence of obesity varied across Hispanic/Latino backgrounds, with the highest prevalence observed among women and men of Puerto Rican background and the lowest among individuals of South American background (Figure 1). Women and men of Puerto Rican background also had the highest prevalence of extreme obesity, with almost double the prevalence rates seen in their counterparts.

3.2. The Association of Sociodemographic and Acculturation Measures with Overweight and Obesity. Table 1 presents results from the generalized logit model that examined whether the differences in BMI categories were due to differences across Hispanic/Latino backgrounds or to differences across field centers. Individuals of Puerto Rican background living in the Bronx were more likely to have extreme obesity, compared to Puerto Ricans living in Chicago, but moderate obesity was similar in these two groups. Individuals of Mexican background living in San Diego were less likely to have moderate obesity than those living in the Bronx but were more likely to have extreme obesity. Individuals of South American background in the Bronx were more likely to be overweight and have moderate obesity than those living in Miami. Among individuals of Central American background, obesity did not vary across field centers.

Household income and educational attainment were not associated with moderate obesity. However, individuals with annual household income $<\$ 40,000$ were more likely to have extreme obesity compared to participants with annual income $>\$ 40,000$. US born participants and those living in the US for 20 or more years were more likely to have moderate obesity than those who had moved to the US less than 20 years ago. This association was much stronger for extreme obesity (Table 1) and was consistent across all Hispanic/Latino background groups (Figure 2). SASH language or the SASH social relations subscales were not associated with moderate or extreme obesity. However, higher social acculturation was inversely associated with overweight (Table 1). Dietary acculturation was not related to overweight or moderate obesity. However, individuals reporting eating Hispanic or American foods in equal amounts were more likely to have extreme obesity, compared to individuals eating mostly Hispanic foods.

Among the foreign-born, individuals who moved to the US when they were younger than 14 years old were more likely to have moderate $(\mathrm{OR}=1.5 ; 95 \% \mathrm{CI} 1.1,1.9)$ and extreme obesity $(\mathrm{OR}=2.2 ; 95 \%$ CI $1.4,3.5)$ than individuals immigrating at an older age; these results were adjusted for 


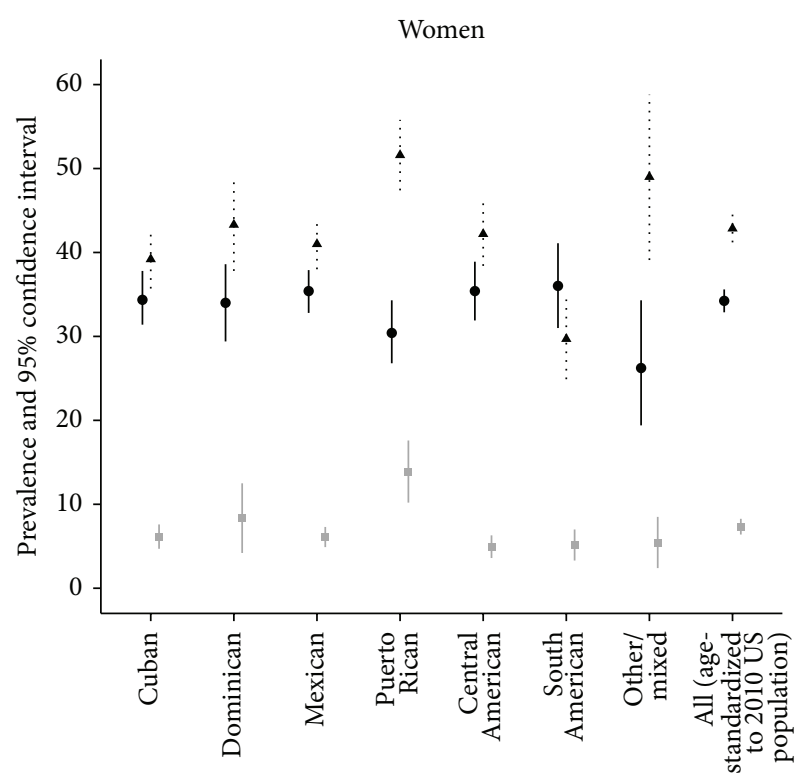

Hispanic/Latino background

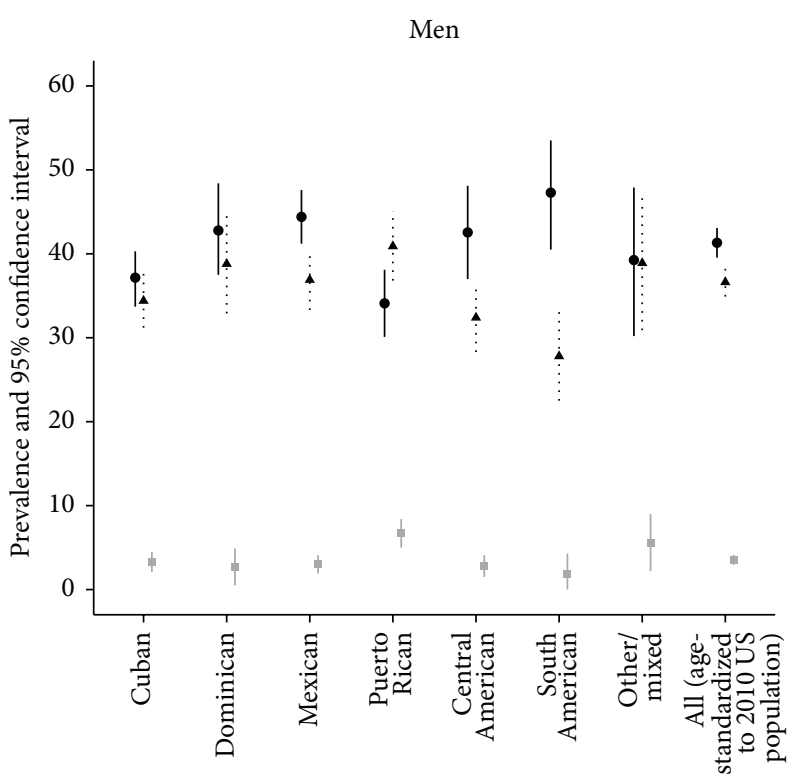

Hispanic/Latino background

$$
\begin{aligned}
& \text { —- Overweight } \\
& \ldots \text {... Obesity } \\
& \ldots \text { Extreme obesity }
\end{aligned}
$$

FIGURE 1: Age adjusted prevalence of overweight $\left(\mathrm{BMI}=25-29 \mathrm{~kg} / \mathrm{m}^{2}\right)$, obesity $\left(\mathrm{BMI} \geq 30 \mathrm{~kg} / \mathrm{m}^{2}\right)$, and extreme obesity $\left(\mathrm{BMI} \geq 40 \mathrm{~kg} / \mathrm{m}^{2}\right)$ by sex and Hispanic/Latino background.

age, sex, Hispanic/Latino background, field center, household income, and educational attainment.

\section{Discussion}

The HCHS/SOL study found a high prevalence of obesity among adults aged 18-74 years. The age-standardized prevalence rates of obesity ( $42.9 \%$ in women and $36.6 \%$ in men) and extreme obesity (7.3\% in women and 3.5\% in men) were similar to estimates reported by NHANES 2009-2010 for Hispanic women (41.4\% obesity and 6\% extreme obesity) and men (37\% obesity and $4.1 \%$ extreme obesity) [1]. The obesity prevalence for Puerto Ricans in HCHS/SOL exceeded these national estimates. Furthermore, the prevalence of extreme obesity among women and men of Puerto Rican background was almost twice the national estimates. This is particularly alarming as extreme obesity is associated with increased morbidity and mortality [8]. Although women and men of South American background had the lowest rates of obesity, the prevalence of overweight among them was comparable to the rates of overweight in the other Hispanic/Latino groups. These findings confirm that obesity is a major public health problem in the US Hispanic/Latino population and has implications for future health care costs given the association between obesity and increased risk of diabetes and cardiovascular disease $[11,14]$.

The study also showed that the prevalence of obesity and extreme obesity varied by field center and that some of the differences in Hispanic/Latino backgrounds could be explained by differences in field centers. Hispanic/Latino background differences were similar across field centers, except for the Bronx. Puerto Ricans living in the Bronx were more likely to have extreme obesity than Puerto Ricans from Chicago. South Americans from the Bronx were more likely to be overweight and have moderate obesity than those from Miami. Individuals of Mexican background in the Bronx were more likely to have moderate obesity but less likely to have extreme obesity, when compared to Mexicans from San Diego. These findings suggest that, at least for some Hispanic/Latino groups, place matters in terms of obesity risk. For example, the Bronx is one of the poorest counties in the state of New York, ranking high in food insecurity in national surveys [15]; low density of food stores and high proportion of unhealthy food outlets also have been reported for the Bronx [16]. Thus, the social and built environment characteristics of the Bronx may explain why certain groups have a higher burden of moderate and extreme obesity.

Consistent with previous studies, we observed that Hispanics/Latinos who were born in the US [4, 17], had lived longer in the US [18-23], or arrived in the US at an early age had the highest prevalence of obesity [20] and extreme obesity. These findings are also consistent with literature involving non-Hispanic immigrants [24]. The high prevalence of obesity among Hispanics/Latinos who have been living in the US for more than 20 years raises questions about the social and environmental conditions impacting risk. These findings suggest that prolonged exposure to the obesogenic US environment, which is conducive of diets rich in energy dense foods and low physical activity, plays a greater 
TABLE 1: Adjusted odds ratio $(\mathrm{OR})^{*}$ for the association of moderate and extreme obesity with Hispanic background, sociodemographic variables, and acculturation.

\begin{tabular}{|c|c|c|c|}
\hline & $\begin{array}{c}\text { Overweight } \\
\left(\mathrm{BMI}=25-29.9 \mathrm{~kg} / \mathrm{m}^{2}\right) \\
\text { OR }(95 \% \mathrm{CI})\end{array}$ & $\begin{array}{c}\text { Moderate obesity } \\
\left(\mathrm{BMI}=30-39 \mathrm{~kg} / \mathrm{m}^{2}\right) \\
\text { OR }(95 \% \mathrm{CI})\end{array}$ & $\begin{array}{c}\text { Extreme obesity } \\
\left(\mathrm{BMI} \geq 40 \mathrm{~kg} / \mathrm{m}^{2}\right) \\
\text { OR }(95 \% \mathrm{CI})\end{array}$ \\
\hline \multicolumn{4}{|l|}{ Hispanic/Latino background } \\
\hline \multicolumn{4}{|l|}{ Central-American } \\
\hline Bronx versus Chicago & $1.07(0.56,2.04)$ & $1.40(0.76,2.59)$ & $1.06(0.40,2.81)$ \\
\hline Miami versus Chicago & $0.99(0.64,1.54)$ & $1.33(0.92,1.93)$ & $1.52(0.75,3.07)$ \\
\hline Bronx versus Miami & $1.07(0.58,1.98)$ & $1.05(0.60,1.85)$ & $0.70(0.29,1.69)$ \\
\hline \multicolumn{4}{|l|}{ Mexican } \\
\hline Bronx versus Chicago & $1.42(0.83,2.43)$ & $1.66(0.94,2.93)$ & $0.13(0.03,0.58)$ \\
\hline Chicago versus San Diego & $1.06(0.86,1.31)$ & $1.30(1.02,1.64)$ & $1.40(0.95,2.07)$ \\
\hline San Diego versus Bronx & $0.66(0.38,1.15)$ & $0.47(0.26,0.83)$ & $5.48(1.23,24.50)$ \\
\hline \multicolumn{4}{|l|}{ Puerto Rican } \\
\hline Bronx versus Chicago & $1.39(0.97,2.01)$ & $1.22(0.83,1.80)$ & $1.78(1.04,3.06)$ \\
\hline \multicolumn{4}{|l|}{ South American } \\
\hline Bronx versus Chicago & $1.31(0.67,2.57)$ & $1.32(0.63,2.76)$ & $2.49(0.78,7.90)$ \\
\hline Chicago versus Miami & $1.60(1.02,2.51)$ & $1.62(0.96,2.75)$ & $0.66(0.19,2.22)$ \\
\hline Bronx versus Miami & $2.10(1.05,4.18)$ & $2.15(1.06,4.37)$ & $1.63(0.51,5.26)$ \\
\hline \multicolumn{4}{|l|}{ Household income } \\
\hline Missing & $0.77(0.60,0.99)$ & $0.81(0.62,1.06)$ & $0.82(0.53,1.29)$ \\
\hline$\leq \$ 20,000$ & $1.05(0.87,1.28)$ & $1.13(0.92,1.38)$ & $1.62(1.17,2.26)$ \\
\hline$\$ 20,001-\$ 40,000$ & $0.99(0.81,1.21)$ & $1.10(0.89,1.36)$ & $1.65(1.16,2.34)$ \\
\hline$>\$ 40,000$ & Reference & Reference & Reference \\
\hline \multicolumn{4}{|l|}{ Educational attainment } \\
\hline Less than high school education & $1.10(0.93,1.32)$ & $1.19(1.00,1.42)$ & $1.21(0.90,1.62)$ \\
\hline High school education or equivalent & $1.07(0.91,1.25)$ & $1.10(0.93,1.31)$ & $1.30(0.95,1.77)$ \\
\hline Greater than high school education & Reference & Reference & Reference \\
\hline \multicolumn{4}{|l|}{ Number of years living in the US } \\
\hline Less than 20 years & Reference & Reference & Reference \\
\hline$\geq 20$ years & $0.98(0.82,1.17)$ & $1.30(1.10,1.54)$ & $2.12(1.59,2.83)$ \\
\hline Born mainland US & $0.91(0.72,1.16)$ & $1.30(1.00,1.69)$ & $2.77(1.88,4.08)$ \\
\hline \multicolumn{4}{|l|}{ Acculturation (SASH) } \\
\hline Language acculturation & $1.03(0.94,1.14)$ & $1.06(0.94,1.18)$ & $1.16(1.00,1.35)$ \\
\hline Social relations & $0.87(0.77,0.99)$ & $0.91(0.80,1.05)$ & $0.95(0.78,1.17)$ \\
\hline \multicolumn{4}{|l|}{ Type of food eaten (dietary acculturation) } \\
\hline Mainly Hispanic & Reference & Reference & Reference \\
\hline Mostly Hispanic & $1.05(0.91,1.23)$ & $0.96(0.83,1.10)$ & $1.22(0.93,1.60)$ \\
\hline Equal amounts & $1.03(0.84,1.27)$ & $1.07(0.89,1.30)$ & $1.45(1.04,2.02)$ \\
\hline Mainly/mostly American & $0.88(0.61,1.27)$ & $1.03(0.71,1.50)$ & $0.55(0.31,0.95)$ \\
\hline
\end{tabular}

${ }^{*}$ From generalized logistic regression model adjusted for all covariates listed in the table plus sex, age group, and sampling weights.

role in explaining excess weight among Hispanics/Latinos than acculturation per se, a point that has been made by others $[4,25,26]$. It is possible that longer duration of residency allows for more time for social and environmental factors to influence changes in behaviors and risk of obesity [5]. It is also important to note that this pattern of more obesity with longer duration of residency in the US was consistent across Hispanic/Latino backgrounds; as such, it reinforces the notion that longer exposure to an obesogenic environment is widespread across the country. However, as these analyses were cross-sectional, we must interpret results with caution. This limitation may be relevant as the association of length of residency in the US with obesity may be due to secular trends of increasing weight affecting all segments of the population and not the duration of residency per se [27]. 


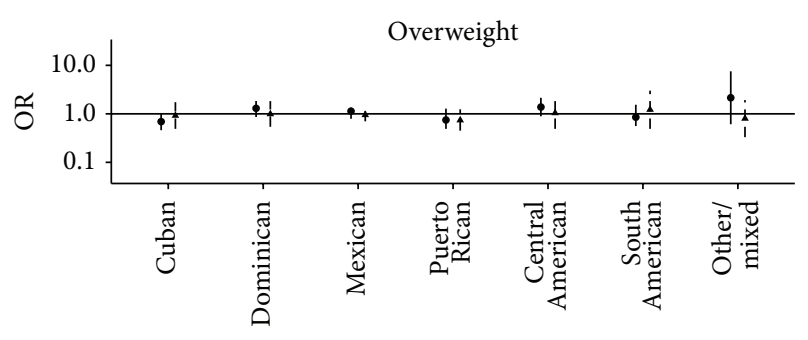

Hispanic/Latino background

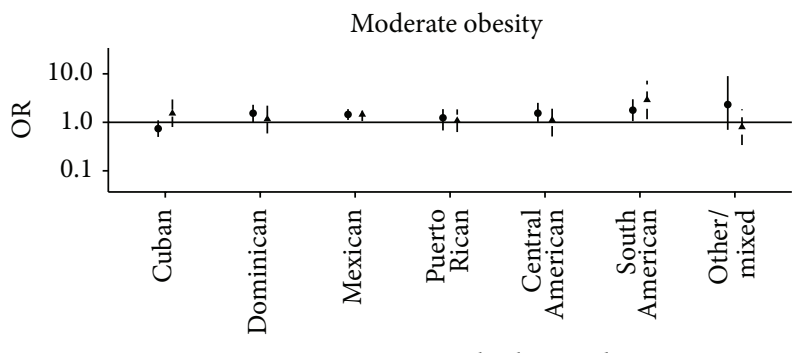

Hispanic/Latino background

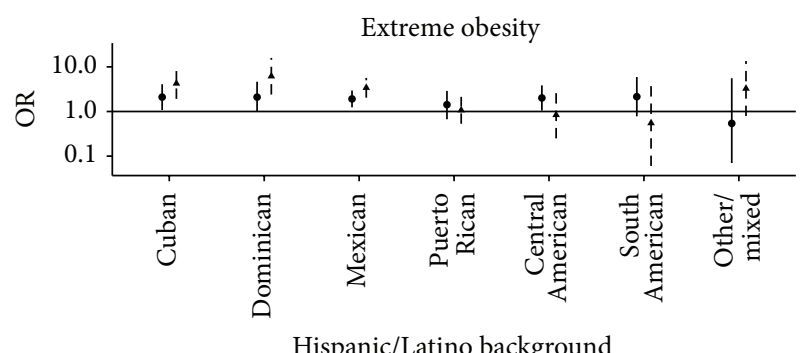

Hispanic/Latino background

$\rightarrow \geq 20$ years in US versus $<20$ years in US

- . - US born versus $<20$ years in US

FIGURE 2: Odds ratio (OR) for the association of years living in the US with overweight $\left(\mathrm{BMI}=25-29.9 \mathrm{~kg} / \mathrm{m}^{2}\right)$, moderate obesity $\left(\mathrm{BMI}=30-39 \mathrm{~kg} / \mathrm{m}^{2}\right)$, and extreme obesity $\left(\mathrm{BMI} \geq 40 \mathrm{~kg} / \mathrm{m}^{2}\right)$ by Hispanic/Latino background. Models are adjusted for age, sex, household income, educational attainment, acculturation field center, and sampling weights. Reference category is living in the US for $<20$ years.

Among the foreign-born, those who had immigrated to the US before the age of 14 years were more likely to have moderate and extreme obesity compared to participants who moved to the US in their twenties or older. This information supports the growing body of evidence regarding the health risks faced by "Generation 1.5" [2], those who immigrate before the age of 14 . More research is needed on how to prevent further escalation of weight and associated health consequences in this population.

This study did not find an association of obesity with acculturation, as measured by the SASH language and social relations subscales. The process of acculturation is complex, and there is as yet no consensus on the best approach for comprehensive assessments of acculturation in epidemiological studies [28-30]. HCHS/SOL used a short scale whose constructs assess language acculturation and ethnic social relations (social acculturation), but it did not capture biculturalism. It is possible that a more refined measure of acculturation that could identify people as bicultural or with different degrees of acculturation across other domains (e.g., exposure to media) could reveal different patterns of association. Dietary acculturation was not associated with overweight or moderate obesity, but individuals who reported eating equal amounts of Hispanic and American foods were more likely to have extreme obesity than individuals who reported eating mainly Hispanic foods. This finding is interesting and warrants further exploration, but it is also difficult to interpret given the simplicity of the measure used. Examining detailed dietary patterns to understand what dietary choices people make in the process of acculturation is an area that needs to be examined. Furthermore, examining the environmental characteristics of the communities in which these participants live may shed light on the processes that put minority and low socioeconomic status populations at risk for obesity. Limited access to healthy foods and safe places to exercise while living in socioeconomically deprived areas are possible explanations for excess weight gain among low income individuals [31]. In addition, recent studies suggest that living in communities that are ethnic enclaves or ethnically isolated may play an important role [32, 33], which merits further exploration as obesity may spread through social networks [34]. The extent to which these social and environmental factors are relevant for acculturation research in Hispanics remains to be examined. Future longitudinal analyses of the HCHS/SOL data will contribute important information on the causal relationships between health behaviors and obesity risk, as well as the extent to which immigration and acculturation variables moderate this relationship.

\section{Disclaimer}

Carmen R. Isasi and Daniela Sotres-Alvarez had full access to the study data and take responsibility for the integrity of the data and accuracy of analyses.

\section{Conflict of Interests}

The authors do not have any conflict of interests to declare.

\section{Authors' Contribution}

All authors were involved in writing the paper and had final approval of the submitted and published versions.

\section{Acknowledgments}

The authors thank the staff and participants of HCHS/SOL for their important contributions. A complete list of staff and investigators has been provided by Sorlie et al. in Ann Epidemiol. 2010 Aug; 20: 642-649 and is also available on the study website https://www.cscc.unc.edu/hchs/. The Hispanic Community Health Study/Study of Latinos was carried out as a collaborative study supported by contracts from the National Heart, Lung, and Blood Institute (NHLBI) to the University of North Carolina (N01-HC65233), University of Miami (N01-HC65234), Albert Einstein College of Medicine 
(N01-HC65235), Northwestern University (N01-HC65236), and San Diego State University (N01-HC65237). The following Institutes/Centers/Offices contributed to the HCHS/SOL through a transfer of funds to the NHLBI: National Institute on Minority Health and Health Disparities, National Institute on Deafness and Other Communication Disorders, National Institute of Dental and Craniofacial Research, National Institute of Diabetes and Digestive and Kidney Diseases, National Institute of Neurological Disorders and Stroke, and NIH Institution-Office of Dietary Supplements.

\section{References}

[1] K. M. Flegal, D. Carroll, B. K. Kit, and C. L. Ogden, "Prevalence of obesity and trends in the distribution of body mass index among US adults, 1999-2010," The Journal of the American Medical Association, vol. 307, no. 5, pp. 491-497, 2012.

[2] Pew Hispanic Center, Hispanics. A People in Motion. A Pew Research Center Project, Pew Hispanic Center, 2005.

[3] A. Tovar, A. Must, N. Metayer et al., "Immigrating to the US: what Brazilian, Latin American and Haitian women have to say about changes to their lifestyle that may be associated with obesity," Journal of Immigrant and Minority Health, vol. 15, no. 2, pp. 357-364, 2013.

[4] M. J. Creighton, N. Goldman, A. R. Pebley, and C. Y. Chung, "Durational and generational differences in Mexican immigrant obesity: is acculturation the explanation?" Social Science \& Medicine, vol. 75, no. 2, pp. 300-310, 2012.

[5] J. Berry, "Conceptual approaches to acculturation," in Acculturation: Advances in Theory, Measurement, and Applied Research, K. Chun, P. B. Organista, and G. Marin, Eds., American Psychological Association, Washington, DC, USA, 2003.

[6] G. X. Ayala, B. Baquero, and S. Klinger, "A systematic review of the relationship between acculturation and diet among Latinos in the United States: implications for future research," Journal of the American Dietetic Association, vol. 108, no. 8, pp. 1330-1344, 2008.

[7] R. Sturm and A. Hattori, "Morbid obesity rates continue to rise rapidly in the United States," International Journal of Obesity, vol. 37, no. 6, pp. 889-891, 2013.

[8] K. McTigue, J. C. Larson, A. Valoski et al., "Mortality and cardiac and vascular outcomes in extremely obese women," Journal of the American Medical Association, vol. 296, no. 1, pp. 79-86, 2006.

[9] P. D. Sorlie, L. M. Avilés-Santa, S. Wassertheil-Smoller et al., "Design and implementation of the Hispanic Community Health Study/Study of Latinos," Annals of Epidemiology, vol. 20, no. 8, pp. 629-641, 2010.

[10] L. M. LaVange, W. D. Kalsbeek, P. D. Sorlie et al., "Sample design and cohort selection in the Hispanic Community Health Study/Study of Latinos," Annals of Epidemiology, vol. 20, no. 8, pp. 642-649, 2010.

[11] National Heart Lung and Blood Institute, Clinical Guidelines on the Identification, Evaluation, and Treatment of Overweight and Obesity in Adults. The Evidence Report, National Institutes of Health, 1998.

[12] G. Marin, F. Sabogal, B. V. Marin, F. Otero-Sabogal, and E. Perez-Stable, "Development of a short acculturation scale for Hispanics," Hispanic Journal of Behavioral Sciences, vol. 9, no. 2, pp. 183-205, 1987.
[13] O. Hellevik, "Linear versus logistic regression when the dependent variable is a dichotomy," Quality and Quantity, vol. 43, no. 1, pp. 59-74, 2009.

[14] R. Sturm, "The effects of obesity, smoking, and drinking on medical problems and costs," Health Affairs, vol. 21, no. 2, pp. 245-253, 2002.

[15] Food Research and Action Center, Food Hardship: A Closer Look at Hunger-Data for the Nation, States, 100 MSAs, and Every Congressional District, Food Research and Action Center, Washington, DC, USA, 2010.

[16] J. H. Stark, K. Neckerman, G. S. Lovasi et al., "Neighbourhood food environments and body mass index among New York City adults," Journal of Epidemiology \& Community Health, vol. 67, no. 9, pp. 736-742, 2013.

[17] M. S. Goel, E. P. McCarthy, R. S. Phillips, and C. C. Wee, "Obesity among US immigrant subgroups by duration of residence," Journal of the American Medical Association, vol. 292, no. 23, pp. 2860-2867, 2004.

[18] G. X. Ayala, J. P. Elder, N. R. Campbell et al., "Correlates of body mass index and waist-to-hip ratio among Mexican women in the United States: implications for intervention development," Women's Health Issues, vol. 14, no. 5, pp. 155-164, 2004.

[19] M. S. Kaplan, N. Huguet, J. T. Newsom, and B. H. McFarland, "The association between length of residence and obesity among Hispanic immigrants," American Journal of Preventive Medicine, vol. 27, no. 4, pp. 323-326, 2004.

[20] P. Gordon-Larsen, K. M. Harris, D. S. Ward, and B. M. Popkin, "Acculturation and overweight-related behaviors among Hispanic immigrants to the US: the National Longitudinal Study of Adolescent Health," Social Science and Medicine, vol. 57, no. 11, pp. 2023-2034, 2003.

[21] D. A. Himmelgreen, R. Pérez-Escamilla, D. Martinez et al., "The longer you stay, the bigger you get: length of time and language use in the U.S. are associated with obesity in Puerto Rican women," The American Journal of Physical Anthropology, vol. 125, no. 1, pp. 90-96, 2004.

[22] A. F. Abraído-Lanza, M. T. Chao, and K. R. Flórez, "Do healthy behaviors decline with greater acculturation? Implications for the Latino mortality paradox," Social Science and Medicine, vol. 61, no. 6, pp. 1243-1255, 2005.

[23] J. V. Bowie, H. S. Juon, J. Cho, and E. M. Rodriguez, "Factors associated with overweight and obesity among Mexican Americans and Central Americans: results from the 2001 California Health Interview Survey," Preventing Chronic Disease, vol. 4, no. 1, p. A10, 2007.

[24] D. S. Barrington, M. C. Baquero, L. N. Borrell, and N. D. Crawford, "Racial/ethnic disparities in obesity among US-born and foreign-born adults by sex and education," Obesity, vol. 18, no. 2, pp. 422-424, 2010.

[25] R. E. Zambrana and O. Carter-Pokras, "Role of acculturation research in advancing science and practice in reducing health care disparities among latinos," The American Journal of Public Health, vol. 100, no. 1, pp. 18-23, 2010.

[26] L. M. Hunt, S. Schneider, and B. Comer, "Should 'acculturation' be a variable in health research? A critical review of research on US Hispanics," Social Science and Medicine, vol. 59, no. 5, pp. 973-986, 2004.

[27] S. S. Albrecht, A. V. D. Roux, N. R. Kandula, T. L. Osypuk, H. Ni, and S. Shrager, "Immigrant assimilation and BMI and waist size: a longitudinal examination among hispanic and chinese participants in the multi-ethnic study of atherosclerosis," Obesity, vol. 21, no. 8, pp. 1695-1703, 2013. 
[28] P. M. Wallace, E. A. Pomery, A. E. Latimer, J. L. Martinez, and P. Salovey, "A review of acculturation measures and their utility in studies promoting Latino health," Hispanic Journal of Behavioral Sciences, vol. 32, no. 1, pp. 37-54, 2010.

[29] G. Marín and R. J. Gamba, "A new measurement of acculturation for hispanics: the bidimensional acculturation scale for hispanics (BAS)," Hispanic Journal of Behavioral Sciences, vol. 18, no. 3, pp. 297-316, 1996.

[30] I. Cuéllar, B. Arnold, and G. González, "Cognitive referents of acculturation: assessment of cultural constructs in Mexican Americans," Journal of Community Psychology, vol. 23, no. 4, pp. 339-356, 1995.

[31] M. Wen and T. N. Maloney, "Latino residential isolation and the risk of obesity in Utah: the role of neighborhood socioeconomic, built-environmental, and subcultural context," Journal of Immigrant and Minority Health, vol. 13, no. 6, pp. 1134-1141, 2011.

[32] M. A. Lee and K. F. Ferraro, "Neighborhood residential segregation and physical health among hispanic Americans: Good, bad, or benign?" Journal of Health and Social Behavior, vol. 48, no. 2, pp. 131-148, 2007.

[33] K. N. Kershaw, S. S. Albrecht, and M. R. Carnethon, "Racial and ethnic residential segregation, the neighborhood socioeconomic environment, and obesity among blacks and Mexican Americans," American Journal of Epidemiology, vol. 177, no. 4, pp. 299-309, 2013.

[34] N. A. Christakis and J. H. Fowler, "The spread of obesity in a large social network over 32 years," The New England Journal of Medicine, vol. 357, no. 4, pp. 370-379, 2007. 


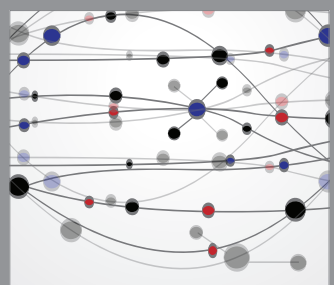

The Scientific World Journal
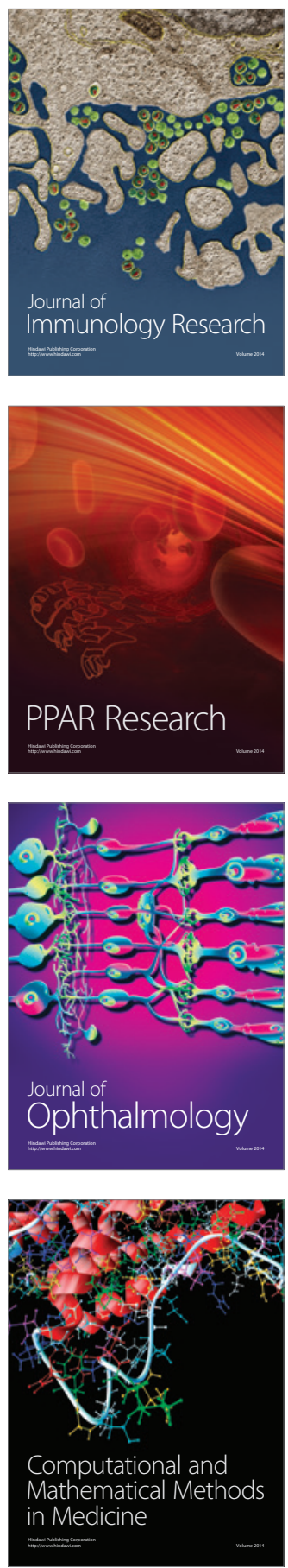

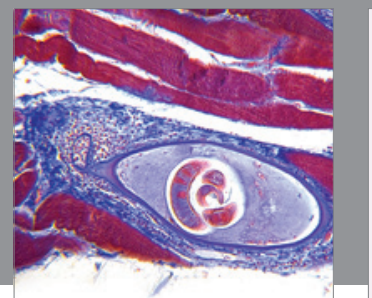

Gastroenterology

Research and Practice
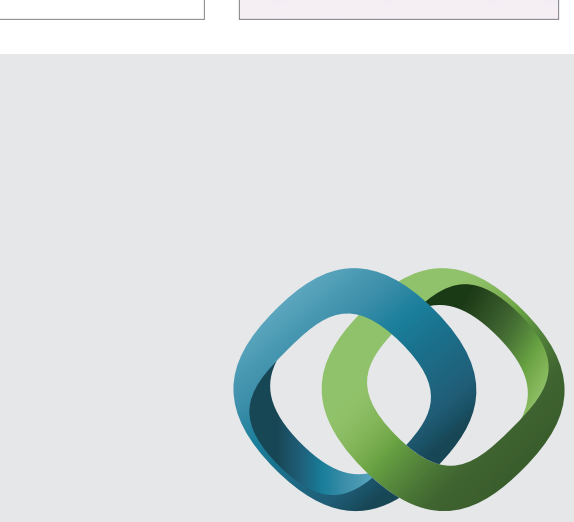

\section{Hindawi}

Submit your manuscripts at

http://www.hindawi.com
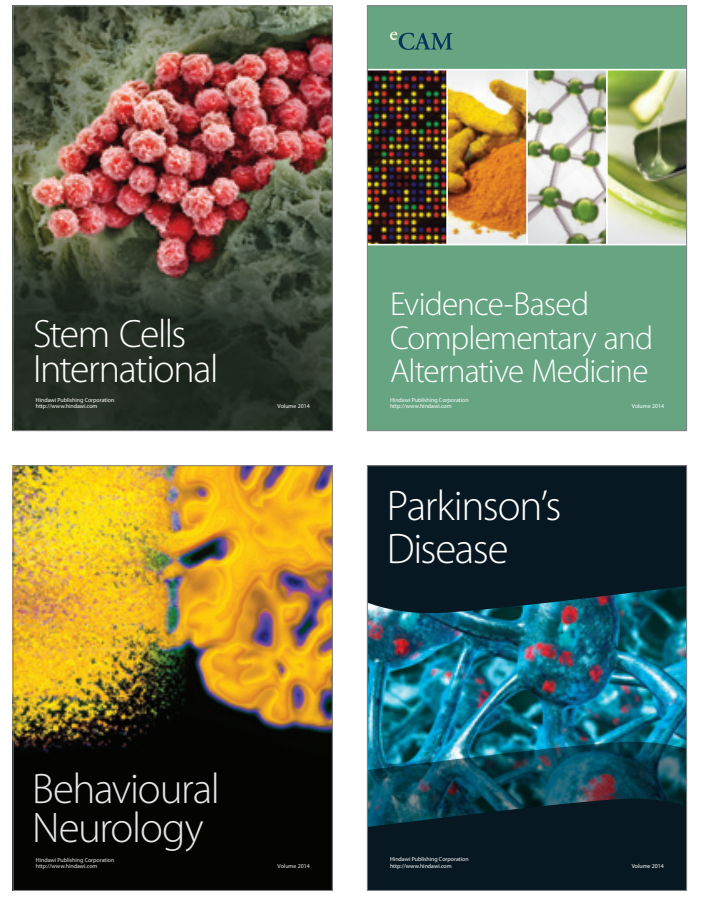
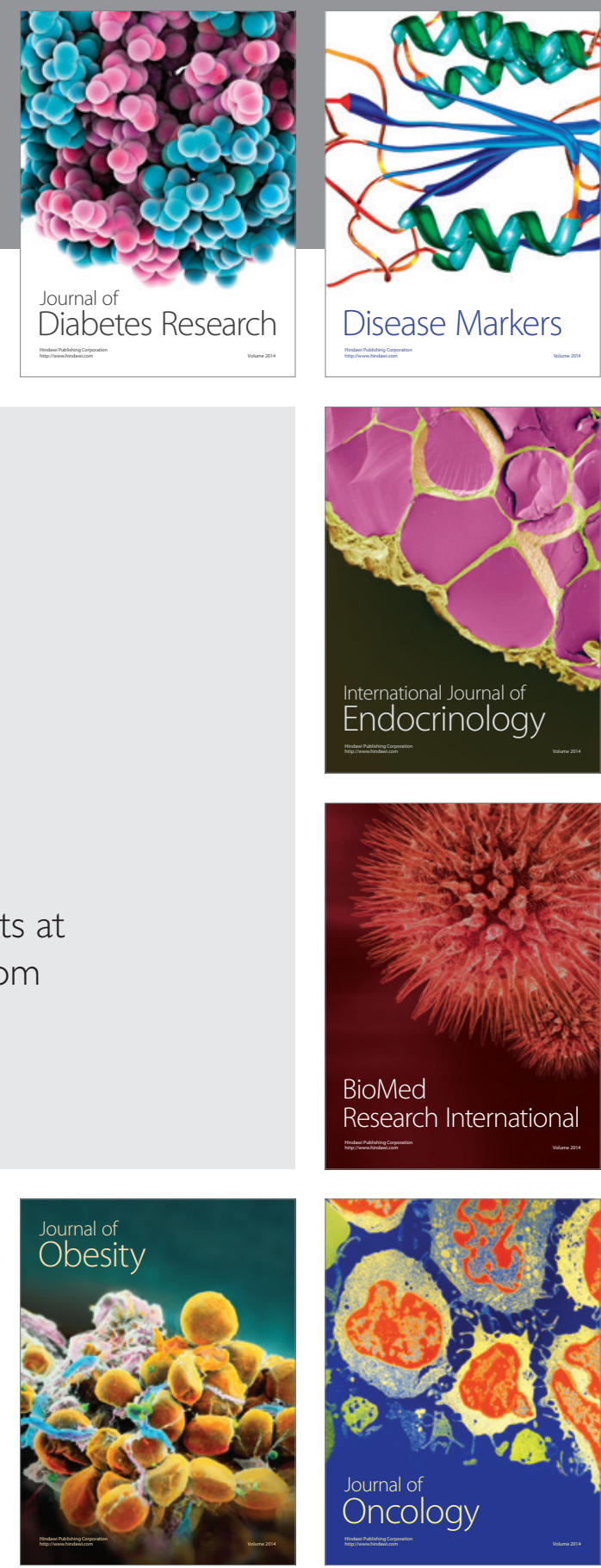

Disease Markers
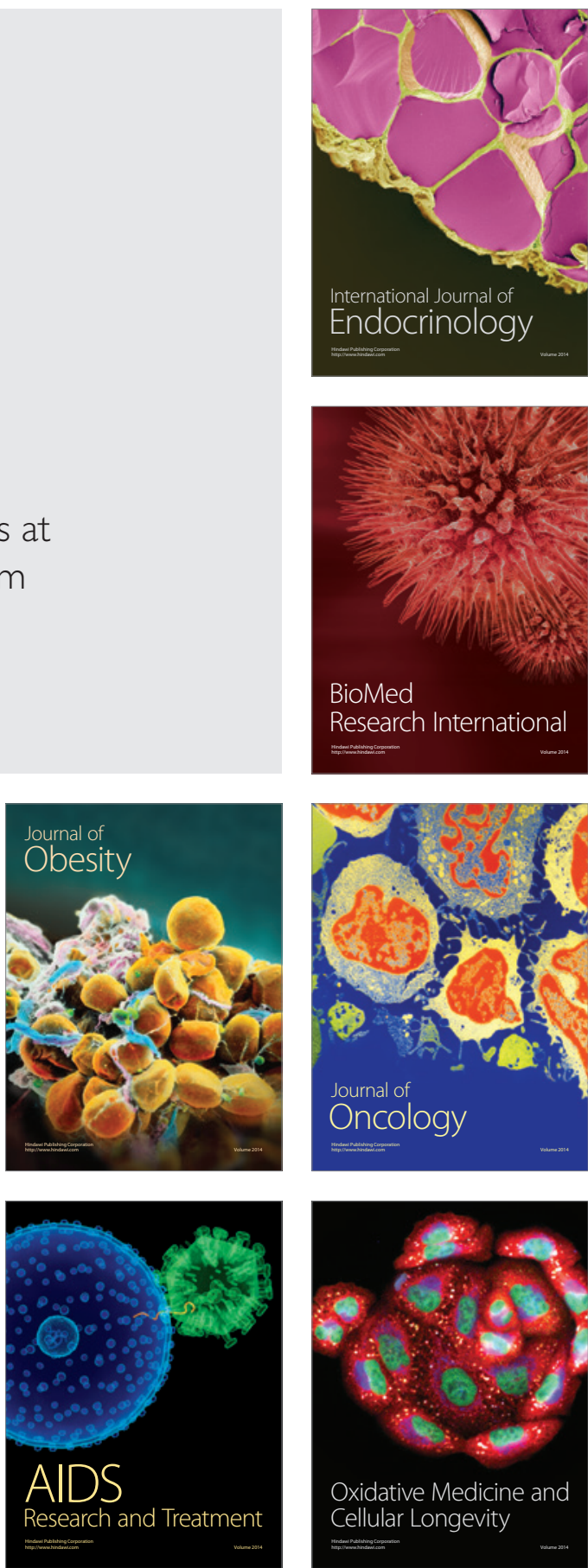